\title{
Smart Polymers in Drug Delivery Applications
}

\section{Geetha B. Heggannavar ${ }^{1, a}$, Divya Achari ${ }^{1, b}$, Cristiana Fernandes ${ }^{2, c}$, Geoffrey Mitchell2,d, Pedro Morouço ${ }^{2, e}$ and Mahadevappa Y. Kariduraganavar ${ }^{1, f^{*}}$}

${ }^{1}$ Department of Chemistry, Karnatak University, Dharwad-580 003, India

${ }^{2}$ CDRSP, Centre for Rapid and Sustainable Product Development, Polytechnic Institute of Leiria, Portugal

ageet.heggannavar@gmail.com, bdivyaacharya1990@gmail.com, ${ }^{\mathrm{C}} \mathrm{cristiana.fernandes@ipleiria.pt,}$ dgeoffrey.mitchell@ipleiria.pt, ${ }^{\mathrm{e} p e d r o . m o r o u c o @ i p l e i r i a . p t, ~}{ }^{\mathrm{f}}$ mahadevappayk@gmail.com

\section{Keywords: Smart polymers, Drug delivery, Pulsatile Systems.}

\begin{abstract}
The most important components of living cells such as carbohydrates, proteins and nucleic acids are the polymeric molecules. Nature utilizes polymers both as constructive elements and as a part of the complicated cell machinery of living things. The rapid advancement in biomedical research has led to many creative applications for biocompatible polymers. With the development of newer and more potent drugs, a parallel expansion in more sophisticated drug delivery systems becomes mandatory. Smart polymeric drug delivery systems can respond to environmental changes and consequently, alter their properties reversibly enabling an efficient and safe drug delivery. This review comprehensively discusses various aspects of these polymers classified in different categories as per the type of stimulus.
\end{abstract}

\section{Introduction}

Life is polymeric in its essence. Throughout our lives, we are constantly dealing with polymers. The most important components of living cells such as carbohydrates, proteins and nucleic acids are the polymeric molecules. Nature utilizes polymers both as constructive elements and as a part of the complicated cell machinery of living things. The phrase smart polymer is appearing with increasing frequency in scientific and engineering publications [1]. Smart polymers are the materials composed of polymers that respond in a dramatic way to very slight changes in their environment. They are also known as 'stimuli responsive polymers' or 'intelligent polymers' or 'environmental-sensitive polymers'. The characteristic feature that makes these polymers 'smart' is their ability to respond to very slight changes in the surrounding environment. The uniqueness of these materials lies not only in the fast microscopic changes occurring in their structure but also these transitions being reversible. The responses are manifested as changes in one or more of the following-shape, surface characteristics, solubility, formation of an intricate molecular assembly, a sol to-gel transition and others. The environmental trigger behind these transitions can be either change in temperature or $\mathrm{pH}$ shift, increase in ionic strength, presence of certain metabolic chemicals, addition of an oppositely charged polymer and polycation polyanion complex formation, changes in electric, magnetic field, light or radiation forces.

Today, the rapid advancement in biomedical research has led to many creative applications for biocompatible polymers. As modern medicine discerns more mechanisms, both of physiology and of pathophysiology, the approach to healing is to mimic, or if possible, to recreate the physiology of healthy functioning. With the development of newer and more potent drugs, a parallel expansion in more sophisticated drug delivery systems becomes mandatory. The new genre of drug delivery systems have advanced features ranging from control drug release to target based expulsion of encapsulated drugs [2]. These attributes convert the simple pharmaceutical formulations into smart drug carrier systems. A variety of strategies have been studied in recent past to impart smart drug delivery features to the pharmaceutical formulations. The most recognized and rational approach to 
achieve this goal can be found by incorporating or embedding in the macromolecular structures called polymers with special capabilities to modulate or moderate the drug release on application of a stimuli [3]. Smart polymeric drug-delivery systems have the ability to respond to environmental changes and consequently, alter their properties reversibly enabling an efficient and safe drug delivery. Fig. 1 depicts various stimuli responsible for controlling drug release from smart polymeric drug delivery systems [4]. This review comprehensively discusses various aspects of these polymers classified in different categories as per the type of stimulus.

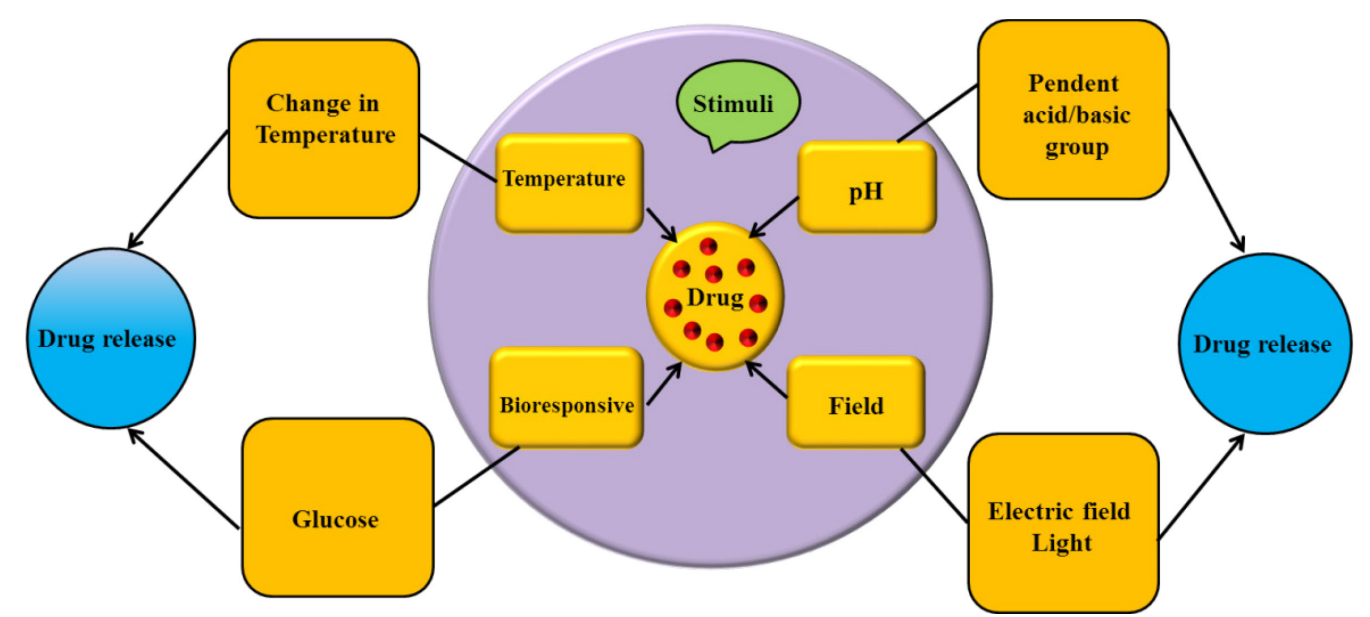

Fig. 1 Various stimuli responsible for controlling the drug release from smart polymeric systems.

Classification of Stimuli Responsive Polymers. The stimuli that can be used for triggering the delivery of drugs, genes, or diagnostic agents from the carriers are diverse. They can be classified into two categories:

(i) Internal stimuli-responsive polymers

(ii) External stimuli-responsive polymers.

Internal stimuli-responsive polymers. Internal stimuli of chemical and biochemical origin include cellular temperature difference, $\mathrm{pH}$-shift, and enzyme over-expression in certain pathological states, and redox state of the specific tissues. Therefore, it is further divided as:

Temperature-responsive polymers. These are the polymers which experience a volume phase transition at a certain temperature and thus a sudden change in solvation state. The unique characteristic of temperature responsive polymer is the critical solution temperature at which the polymer changes phase. Therefore, it is defined as the temperature at which the polymer undergoes the phase transition according to its composition. If the polymer undergoes a phase transition from a soluble state (monophasic) to an insoluble state (biphasic) above the critical temperature, i.e., with increase of temperature, it is characterized as having a lower critical solution temperature (LCST); if transition of polymer occurs from an insoluble state to a soluble state with increasing temperature, it has an upper critical solution temperature (UCST) as depicted in Fig. 2 [5-7]. Temperature is the most widely used stimulus in the synthetic and the bio-inspired stimulus-responsive system [6]. 


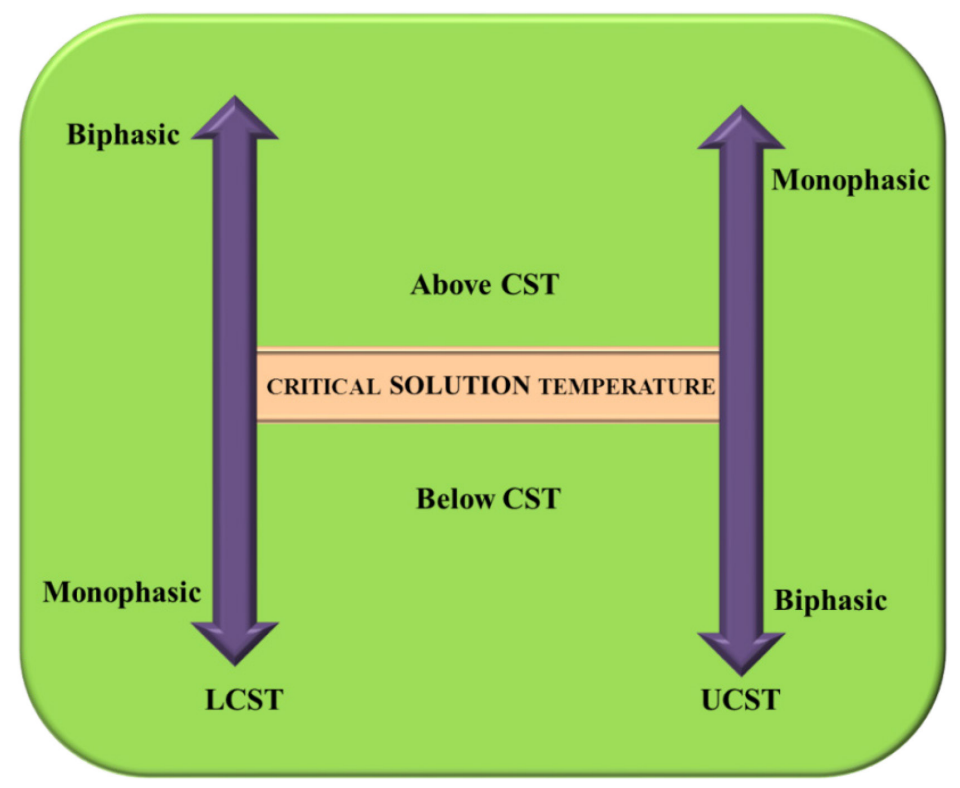

Fig. 2 The behavior of temperature-responsive polymer.

The thermo-responsive polymers have a very common feature which includes the existence of both hydrophobic (e.g., methyl, ethyl, propyl) and hydrophilic (e.g., amide, carboxyl) groups together in one macromolecular network. The polymers with an LCST are mostly used in drug delivery systems. The hydrophobic and the hydrophilic moieties in the molecular chain of a temperatureresponsive polymer define its LCST. Hydrophilic monomers make the LCST increase and even disappear, and hydrophobic ones cause the LCST to decrease. Thus, a proper ratio of hydrophobic or hydrophilic moieties can be incorporated to get a suitable LCST. In case of drug delivery, attaining LCST which is approximate to body temperature is very much essential $[5,8-10]$.

The temperature-responsive polymers are divided into three classes [11]:

a) Polymers based on LCST: These are the polymers that undergo a phase transition from soluble to insoluble state above LCST.

(b) Polymers with amphiphilic balance: This type of amphiphilic block copolymers are reported to have temperature-responsive micellization behavior and form hydrogels above a critical gelation temperature. They can undergo transitions such as a gel-sol phase transition and sol-gel phase transition at $50^{\circ} \mathrm{C}$ and $37^{\circ} \mathrm{C}$ respectively.

(c) Polymers of natural origin: The naturally originated biopolymers such as gellan (composed of glucose and b-d-glucuronic acid and a-l-rhamnose), gelatin, gellan benzyl ester, amylopectin, amylase, and agarose have shown to have temperature-responsive activity and three dimensional chemical structures of some common temperature responsive polymers are presented in Fig. $3[4,8]$.

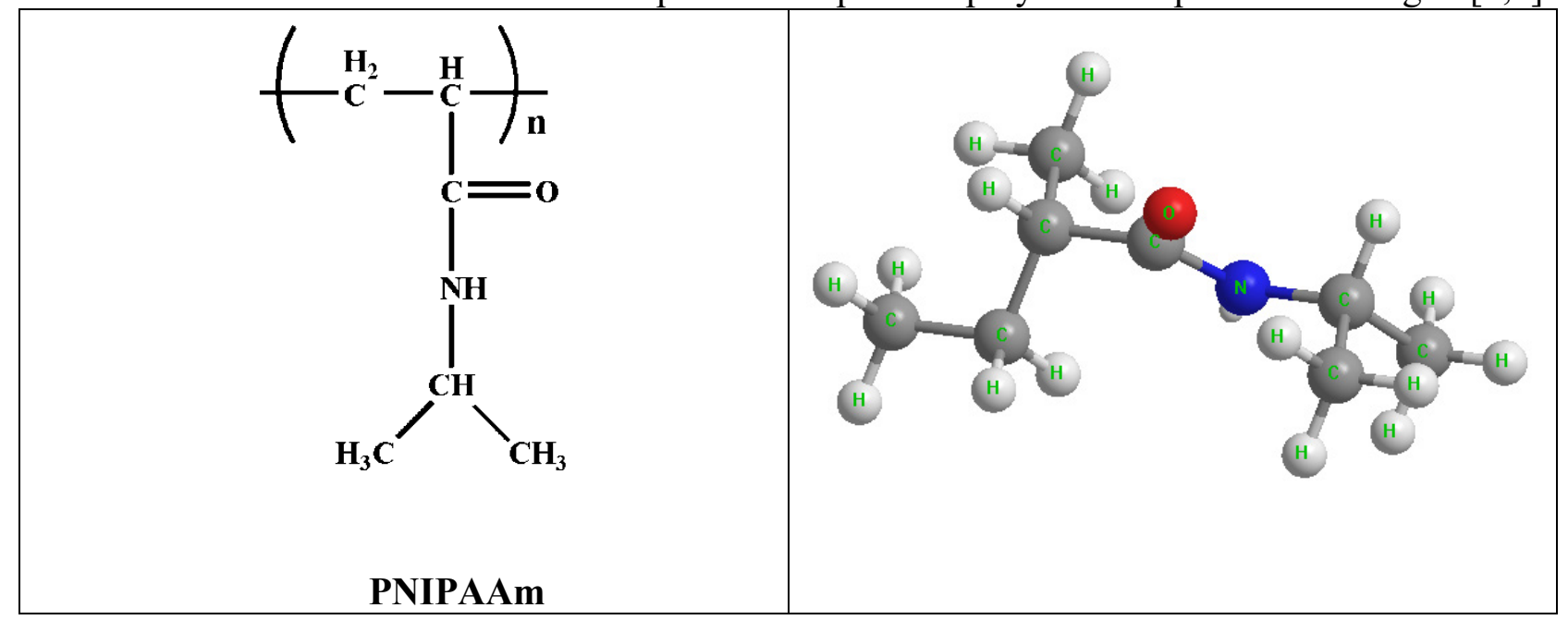




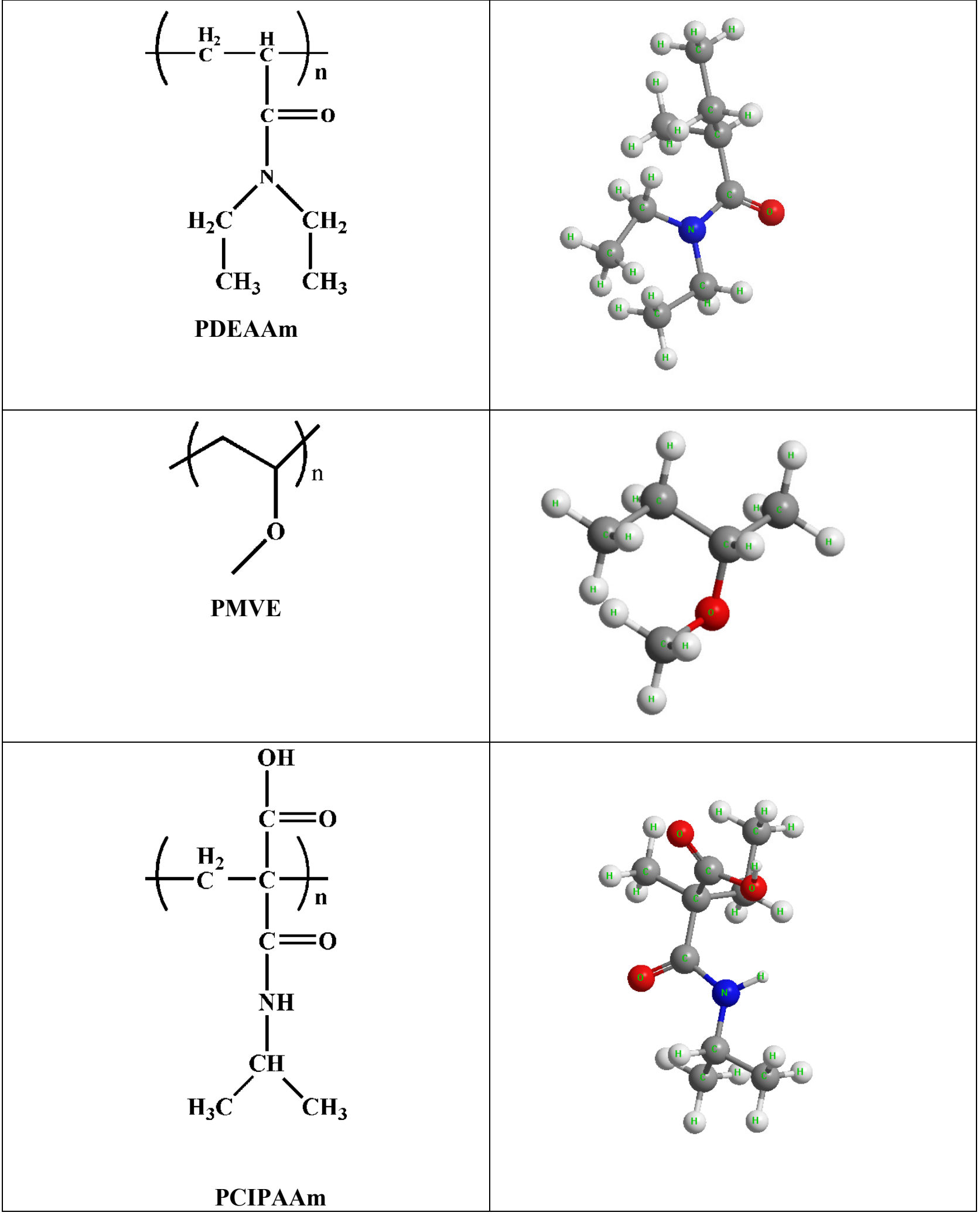




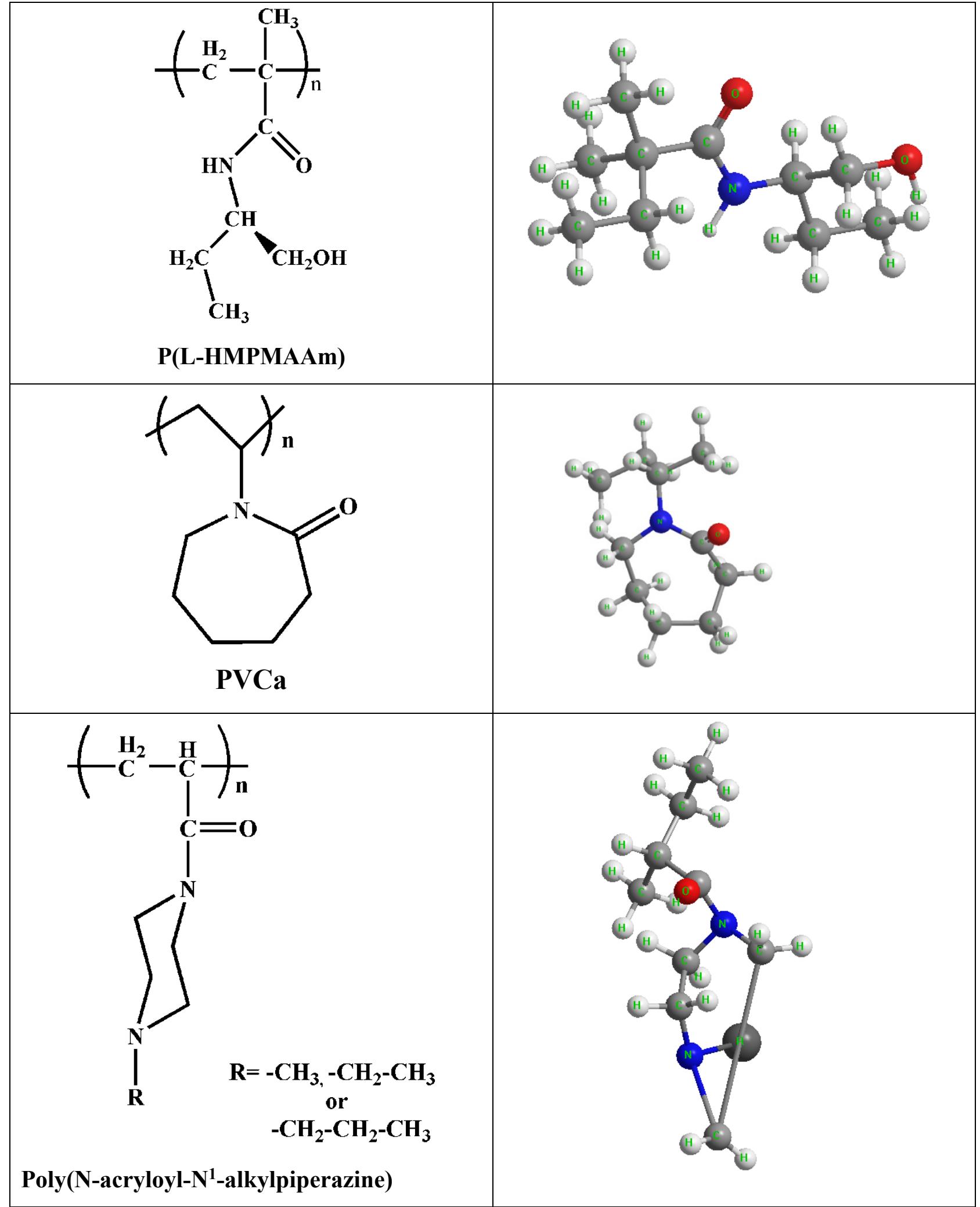

Fig. 3 Illustrations of chemical and three-dimension structures of some common temperature responsive polymers (PNIPAAm, PDEAAm, PMVE, PCIPAAm, P(L-HMPMAAm), PVCa, Poly(N-acryloyl-N1-alkylpiperazine)).

pH-responsive polymers. The $\mathrm{pH}$ is an important signal, as its values vary significantly in different tissues or organs, and in disease states, such as tumorigenesis, infections etc, [12]. These issues can be addressed through $\mathrm{pH}$-responsive materials. Ionizable polymers with a $\mathrm{pKa}$ value between 3 and 10 are candidates for $\mathrm{pH}$-responsive systems and they are among the most studied stimuli-responsive systems [6]. pH-Responsive polymers comprise either polyacids or polybases or 
a combination of both. The chain conformation, solubility, and volume can be manipulated by changes in $\mathrm{pH}$, co-solvent, and electrolytes [12]. The $\mathrm{pH}$-Responsive polymeric systems provide the possibility of fabricating tailorable "smart" functional materials; hence they have found many potential commercial applications, and one among them is in drug delivery system. For example, the extracellular $\mathrm{pH}$ of most tumors is acidic $(\mathrm{pH}$ 5.8-7.2), smart polymeric nanodevices can be designed for anti-cancer drug delivery, where the release of drugs can be triggered by manipulating $\mathrm{pH}$ [13]. The $\mathrm{pH}$-sensitive polymer consists pendant acidic or basic groups, which in response to change in environmental $\mathrm{pH}$, either accept or release a proton [4].

The $\mathrm{pH}$-responsive polymers are classified into four categories:

(a) Weak polyacids: This category of polymers undergoes an ionization/deionization transition from $\mathrm{pH}$ 4-8. The most representative weak polyacids are the one with carboxylic group and with $\mathrm{pKa}$ values of around 5-6. Carboxylic groups in polymers accept protons at low $\mathrm{pH}$, while releasing them at high $\mathrm{pH}$. Therefore, they are transformed into polyelectrolytes at high $\mathrm{pH}$ with electrostatic repulsion forces between the molecular chains [11]. A polymer with polyacidic framework will swell with increasing $\mathrm{pH}$. The changes in polymer structure of polyacid with respect to $\mathrm{pH}$ are illustrated in Fig. 4.

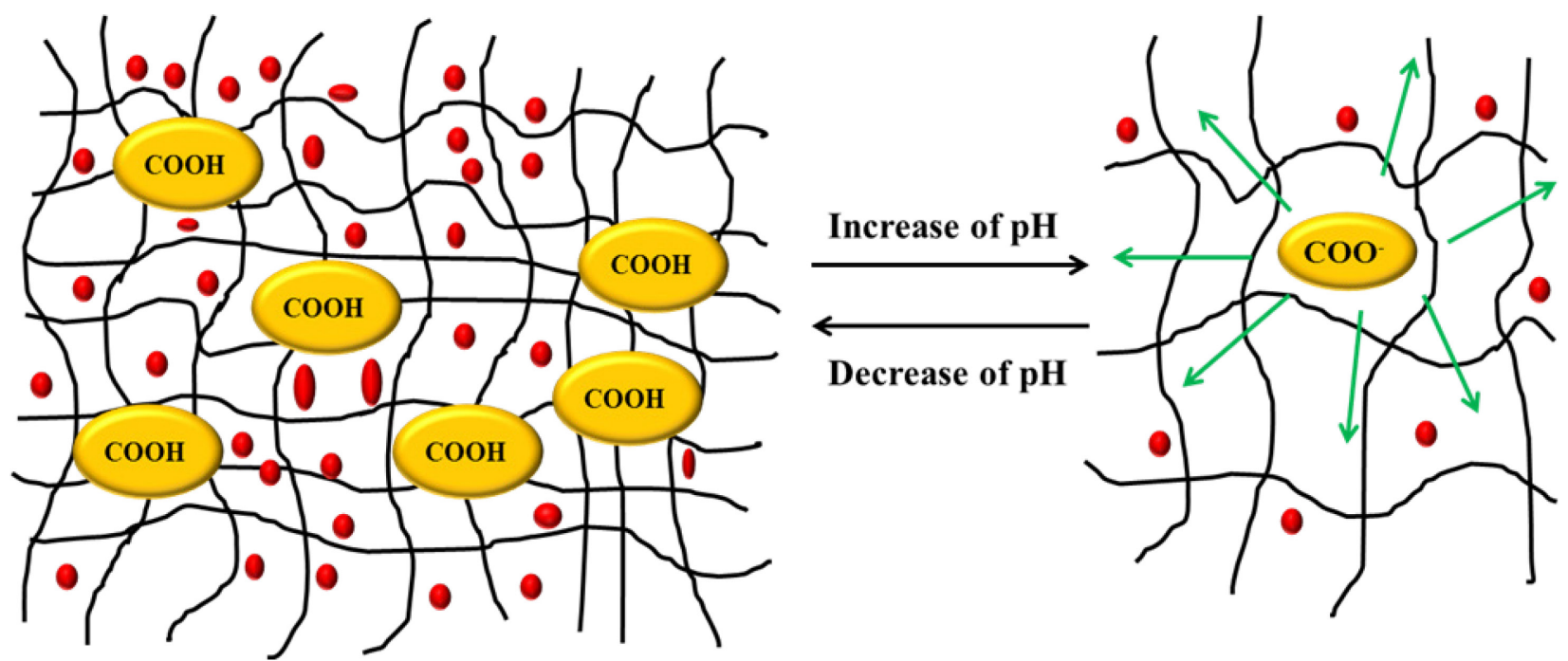

Fig. 4 Schematic representation of changes in polymer structure of polyacids with $\mathrm{pH}$ values [14].

(b) Polybases: Polymers under this category have the amine groups in their side chains. The amine groups gain protons under acidic condition and release them under basic condition as shown in Fig. 5.
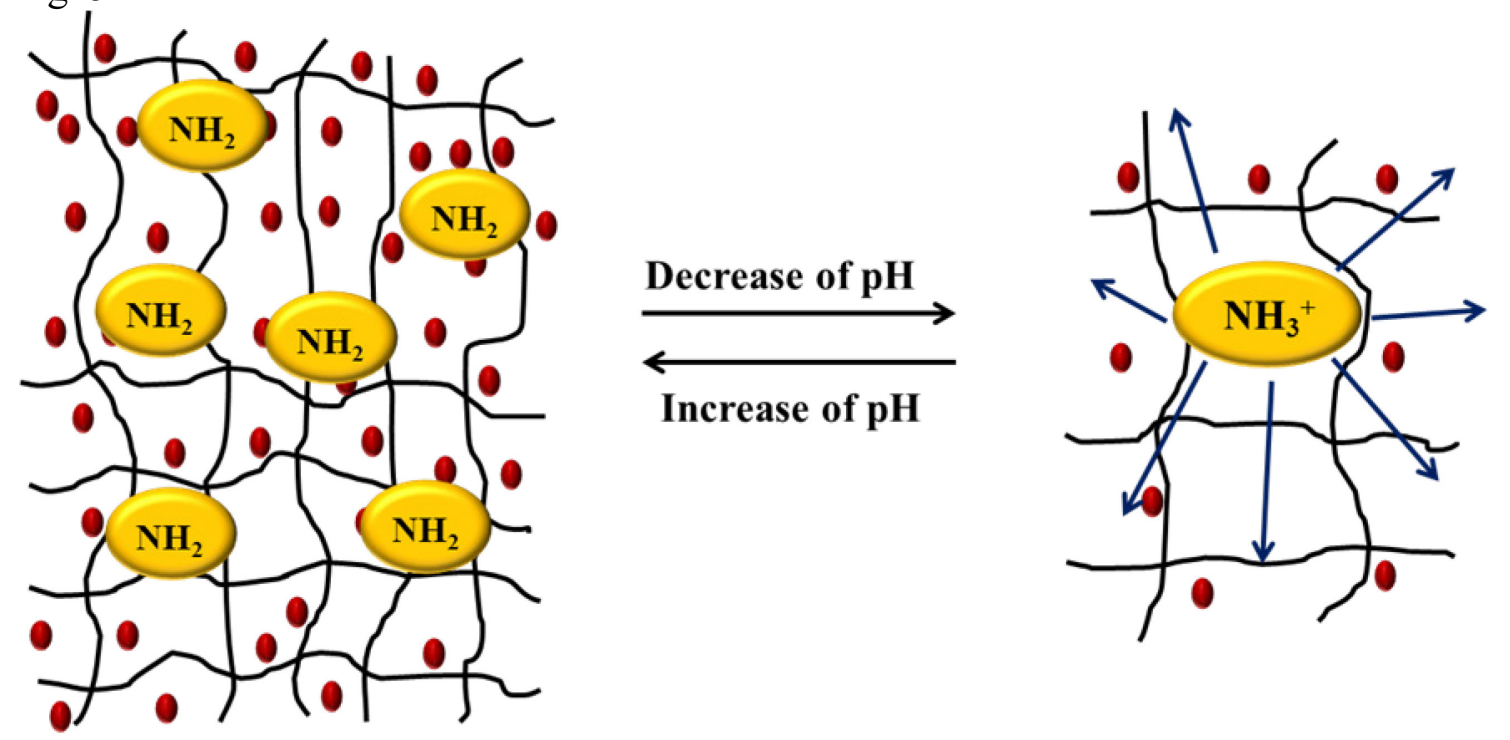

Fig. 5 Schematic representation of changes in polymer structure of polybases with $\mathrm{pH}$ values [14]. 
(c) pH-responsive degradable polymers: These $\mathrm{pH}$-responsive degradable polymers have various acid cleavable covalent links, such as acetal, orthoester, hydrazone, imine, cis-acotinyl, and oxime, which undergo hydrolysis rapidly in the endosomal compartment $(\mathrm{pH} \sim 5)$ [15].

(d) Biopolymers and artificial polypeptides: This type of polymers is weakly ionizable polysaccharides, such as alginate and chitosan, which show $\mathrm{pH}$ responsive phase transition.

Enzyme-responsive polymers. The high specificity of the enzymes is highly exploited by enzymeresponsive polymer systems ensuring that the crosslinks are broken only in the presence of a specific enzyme resulting in site-specific release of the drug. Enzymes are highly selective in their reactivity, they are operable under mild conditions when present in vivo, and are vital components in many biological pathways [16]. They are involved in all biological and metabolic processes, serving as the prime protagonists in the chemistry of living organisms at a molecular level. The integration of enzyme-catalyzed reactions with responsive polymers can broaden the design flexibility and scope of applications by endowing the latter with enhance triggering specificity and selectivity [17]. Enzyme-responsive materials are typically composed of an enzyme-sensitive substrate and another component that directs or controls interactions that lead to macroscopic transitions. The enzymes catalytic action on the substrate can cause changes in the transformation of supramolecular architectures and surface properties [16].

Redox-responsive polymers. The redox reactions are characterized by the transfer of electrons between the chemical species. As electrons are responsible for the formation of covalent bonds, this transfer of electrons simultaneously breaks the existing bonds while generating new ones. Different regions of the body, and even different intracellular compartments, have different redox states. These properties of redox reactions make them an ideal target for a stimuli-responsive drug delivery system. For example, if a redox-responsive chemical group is utilized as a tether for a drug, it will be stable when the redox state is neutral, preventing premature release of drug. By choosing a linker that is sensitive to cleavage in the particular redox state in target tissue, drug release can be localized to that tissue. Redox sensitive drug carriers have drawn increasing interest because they contain redox-sensitive polymers with a high redox potential difference (100-1000 fold) between the reducing intracellular space and the oxidizing extracellular space [18].

External stimuli-responsive polymers. The external stimuli-responsive polymers work on the mechanisms such as irradiation (photo), magnetism, and ultrasound.

Photo-responsive polymers. The light-responsive functional groups on repeating units make polymers light responsive to be used in a number of ways [19]. Photo-responsive polymers are interesting practically as well as academically. On photo-irradiation, photo-responsive polymers change reversibly their physical and/or chemical properties, such as conformation, shape, surface wettability, membrane potential, membrane permeability, $\mathrm{pH}$, solubility, sol-gel transition temperature, and phase separation temperature of the polymer blends [20]. Photo-responsive units attached to the macromolecules respond to light or dark conditions giving reversible variations of their structure [21]. These photo-responsive systems are generally composed of a photosensitive element (photochromic molecule, i.e., chromophore), which captures optical signals and converts them to physicochemical signals, and a second functional element (e.g., a protein domain), which senses the physicochemical signals and exhibits new output functions. The absorbed light activates a chemical transformation in the chromophore (e.g., photoisomerization), which subsequently controls the conformation and/or assembly of the surrounding molecules [22]. These fascinating properties suggest that photo-responsive polymers may become suitable materials for designing the new and better drug delivery systems.

Magnetic-responsive polymers. In recent days of research, the magnetic-responsive materials are the topic of great interest due to their potential breakthrough applications in the biomedical, coatings, microfluidics, and microelectronics fields. Integration of magnetic and polymer materials can be used to obtain composites with exceptional magnetic-responsive features. Magnetic actuation provides unique capabilities as it can be spatially and temporally controlled, and can additionally be operated externally to the system, providing a non-invasive approach to remote control [23]. For in 
vivo applications, the magnetic particles must be coated with a biocompatible polymer during or after the synthesis process to prevent the formation of large aggregates, changes from the original structure and biodegradation when exposed to the biological system. The polymer will also allow binding of drugs by covalent attachment, adsorption or entrapment on the particles [24]. Magnetic particles with appropriate surface coatings of polymers are increasingly being used clinically for various biomedical applications, such as magnetic resonance imaging, hyperthermia, drug delivery, tissue repair, cell and tissue targeting and transfection. Magnetic polymer composites can bind to drugs and an external magnetic field can be applied to trap them in the target site. The site specific drug delivery in the body can be achieved by attaching the targeting molecules, such as proteins or antibodies, at particles surfaces [25].

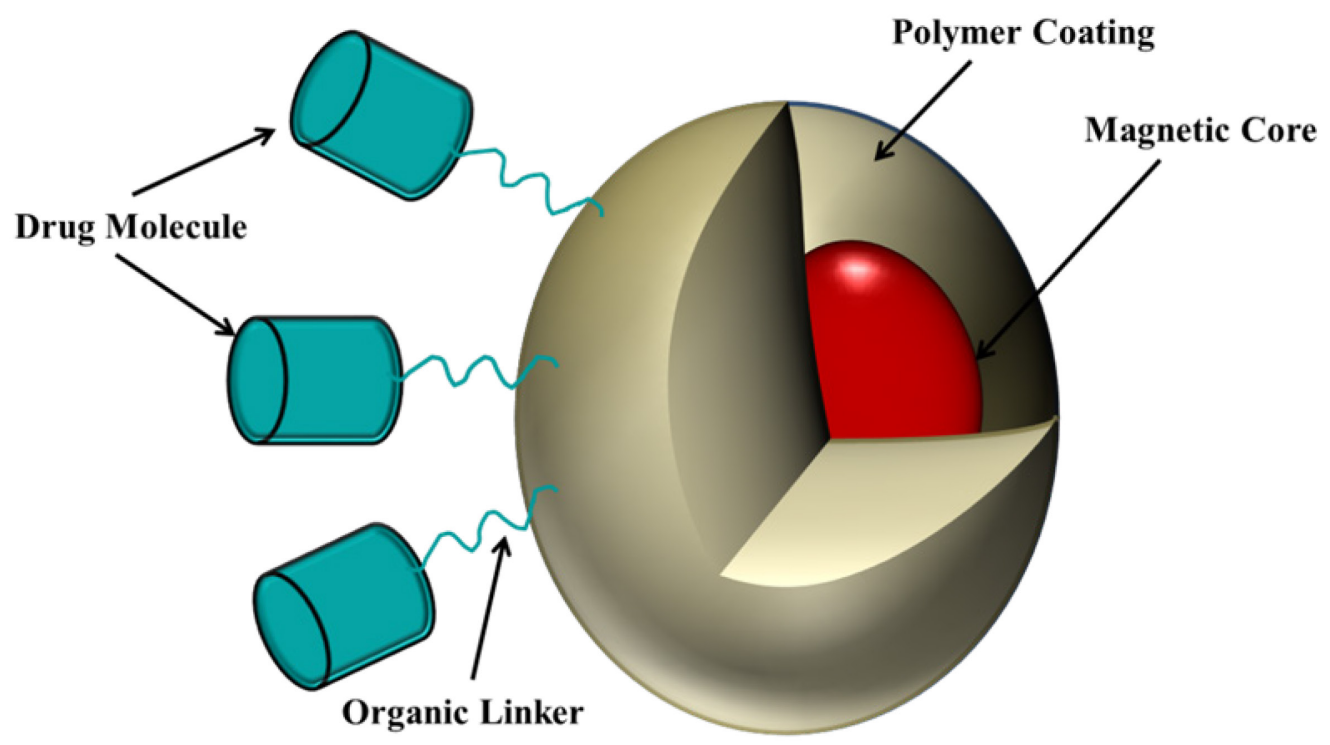

Fig.6 The structure of magnetic-responsive nanocarriers [14].

Ultrasound-responsive polymers. Ultrasound-responsive drug delivery systems have become an important research focus in targeted therapy due to their effectiveness in releasing drug at the specific tissue. Ultrasound impulse is an interesting possibility since it allows spatial and temporal control. It is non-invasive and already widely used and accepted as a biomedical imaging method [26]. High intensity focused ultrasound is an incredibly potent technology for delivering high power densities to controlled, localized areas within the body. Harnessing this localized energy in a manner that triggers controlled reactions could lead to new breakthroughs in the design of novel drug delivery systems. The advantage of ultrasound is that its intensity and depth of focus can be suitably altered to regulate the amount of drug released as well as the location within the biological system.

Smart drug delivery systems. Engineered materials have been explored for developing smart drug delivery systems. Design and multi-functionalities fabricate of efficient smart drug delivery systems are vitally necessary for medicine and healthcare development. In the material science field provides biodegradable, biocompatible, environment-responsive, and highly effective novel polymeric system for targeted delivery. Nanotechnology provides bottom-up and top-down nanofabrication with size controlled and multi-functionality of particulate for targeted delivery. New materials invention and advanced technology have been synergistically achieved in drug delivery so far. The essential goals of medical pharmacology provide the correct medicine, adequate dosage, and proper route at the adequate time to the patient; thus, more research is needed to optimize the therapeutic efficacy of the drug. These are critical principles is behind the smart drug delivery. A smart, controlled delivery system needs synergistic consideration of several factors; these have been summarized in Fig. 7 [27]. 


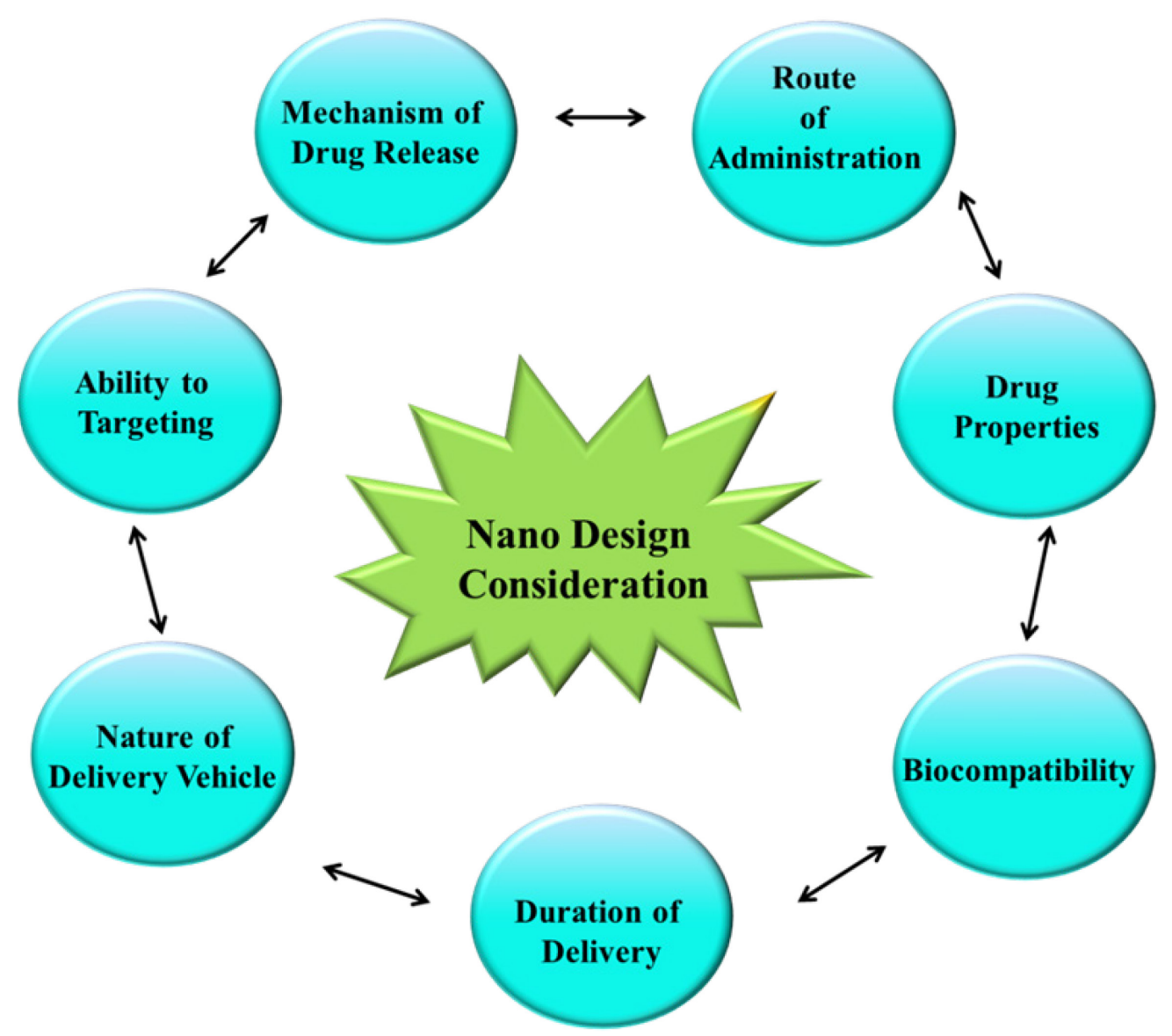

Fig. 7 Requirements of several factors for simultaneous consideration to design a polymeric nanoparticle for the smart drug delivery system.

\section{Classification of Smart Drug-Delivery Systems}

The smart drug-delivery systems can be classified as closed - or open loop systems. Closed-loop systems are also known as environmentally- responsive systems or self-regulated systems in drug delivery application. These self-regulated systems utilize several approaches for the rate control mechanisms, such as thermal, $\mathrm{pH}$-sensitive polymers, enzyme-substrate reactions, $\mathrm{pH}$-sensitive drug solubility, competitive binding, antibody interactions and metal-concentration-dependent hydrolysis. In the controlled drug delivery field, open-loop systems are known as pulsatile or externally regulated. The externally controlled devices apply external triggers for pulsatile delivery such as: ultrasonic, magnetic, electric, light and chemical or biochemical agents. Table 1 summarizes the various smart polymeric drug delivery systems along with their advantages and limitations [4]. 
Table 1 Advantages and limitations of smart polymeric drug delivery systems.

\begin{tabular}{|c|c|c|}
\hline Stimulus & Advantages & Limitations \\
\hline Temperature & $\begin{array}{l}\text { Ease of incorporation of active } \\
\text { moieties inject ability under } \\
\text { application conditions }\end{array}$ & $\begin{array}{l}\text { Simple manufacturing and formulation Low } \\
\text { mechanical strength, biocompatibility issues and } \\
\text { instability of thermolabile drugs }\end{array}$ \\
\hline $\mathrm{pH}$ & Suitable for the & Lack of toxicity data and low mechanical strength \\
\hline Light & $\begin{array}{l}\text { Ease of controlling the trigger } \\
\text { mechanism Accurate control } \\
\text { over the stimulus }\end{array}$ & $\begin{array}{l}\text { Low mechanical strength of gel, chance of } \\
\text { leaching out of noncovalently attached } \\
\text { chromophores }\end{array}$ \\
\hline $\begin{array}{l}\text { Electric } \\
\text { field }\end{array}$ & $\begin{array}{l}\text { Pulsative release with changes } \\
\text { in electric current }\end{array}$ & $\begin{array}{l}\text { Surgical implantation required. Need of an } \\
\text { additional equipment for external application of } \\
\text { stimulus. Difficulty in optimizing the magnitude } \\
\text { of electric current }\end{array}$ \\
\hline Ultrasound & Controllable protein release & $\begin{array}{l}\text { Specialized equipment for controlling the release, } \\
\text { Surgical implantation required for } \\
\text { nonbiodegradable delivery system }\end{array}$ \\
\hline
\end{tabular}

Environmentally-responsive systems. Recently, polymers that alter their characteristics in response to changes in their environment have been of great interest. Several research groups have been developing drug delivery systems, based on these responsive polymers, to mimic the normal physiological process [6,28-30]. In these devices drug delivery is regulated by means of an interaction with the surrounding environment without any external intervention. The most commonly studied polymers, having environmental sensitivity, are either $\mathrm{pH}$ or temperature sensitive. There are also other systems which include specific binding interactions, inflammationsensitive systems and systems utilizing enzymes.

Temperature-sensitive systems. This type of drug delivery systems is based on polymer-water interactions of temperature-sensitive polymers, especially specific hydrophobic/hydrophilic balancing effects, and the configuration of side-groups. When polymer networks swell in a solvent, there is usually a negligible or small positive enthalpy of mixing or dilution. Although a positive enthalpy change opposes the process, the large gain in the entropy drives it. In aqueous polymer solutions the opposite is often observed. This unusual behavior is associated with a phenomenon of polymer phase separation as the temperature is raised to a critical value, known as the lower critical solution temperature (LCST). Polymers characterized by LCST usually shrink, as the temperature is increased through the LCST. Lowering the temperature below LCST, results in the swelling of the polymer. Bio-active agents such as drugs, enzymes, antibodies and genes may be immobilized on or within the temperature sensitive polymers. Wang et al. [31] used the same concept of protein coiledcoil formation and reported on a hybrid system based on water-soluble synthetic polymers and a protein-folding coiled coil. These hydrogels undergo temperature-induced collapse owing to the cooperative conformational transition of the coiled-coil protein domain and may be useful for incorporating and delivering therapeutic proteins. Recently, drug/genes release patterns regulated by environmental temperature changes have been demonstrated by several groups $[6,29,30,32,33]$.

pH-sensitive systems. The $\mathrm{pH}$-sensitive system depends on the $\mathrm{pH}$ range of fluids in various segments of the gastrointestinal tract which provide environmental stimuli for responsive drug release. Studies by several research groups $[28,30,32]$ have been performed on polymers containing weakly acidic or basic groups in the polymeric backbone. The charge density of the polymers depends on $\mathrm{pH}$ and ionic composition of the outer solution (the solution into which the polymer is exposed). Altering the $\mathrm{pH}$ of the solution will cause swelling or deswelling of the polymer. Lowman and Peppas [34] proposed $\mathrm{pH}$ responsive drug delivery based on a complexation-decomplexation 
mechanism. Copolymer networks of poly(methacrylic acid) grafted with poly(ethylene glycol) exhibit $\mathrm{pH}$-dependent swelling behavior due to the reversible formation/dissociation of interpolymer complexes. Drug-delivery systems based on this copolymer showed $\mathrm{pH}$-dependent drug delivery rates.

Protein-sensitive systems. These are the systems which utilize the antibody interactions. Pitt et al. [35] proposed of utilizing morphine (hapten)-antibody interactions to suppress the enzymatic degradation and permeability of polymeric reservoirs or matrix drug-delivery systems. The delivery device consisted of the drug naltrexone contained in a polymeric reservoir or dispersed in an erodible polymeric matrix configuration. The device was coated by covalently grafting morphine to the surface. Exposure of the grafted surface to morphine antibodies resulted in coating of the surface by the antibodies, a process that can be reversed by exposure to exogenous morphine. The presence of the antibodies on the device surface will impede the release of the drug when the mechanism of drug release is dependent on either (a) diffusion through the bulk orvia micropores in the reservoir device membrane, or (b) enzyme-catalyzed surface erosion of the antibodies on the surface or in the pores.

\section{Pulsatile Systems}

The pulsatile systems can be generally triggered ultrasonically, magnetically and electrically.

Ultrasonically stimulated systems. The drug release rates can be repeatedly modulated from a position external to the delivery system by ultrasonic irradiation [36]. When bio erodible polymers were used as the drug-carrier matrices, both the polymer erosion and drug release rates were enhanced when samples were exposed to ultrasound. The system's response to the ultrasonic triggering was rapid and reversible. The releasing agents, p-nitroaniline, p-aminohippurate, bovine serum albumin and insulin, were tested for integrity following exposure to ultrasonic energy, and were found to be intact. Enhanced drug-release rate was also observed when non-erodible polymeric systems were exposed to ultrasound [36,37]. Aschkenasy and Kost [37] showed that poly(ethylenevinyl acetate) (EVAc) matrices, containing low loading of water-soluble particles, swell intensely after immersion in water. This is caused by the osmotic force of the isolated particles which causes the water to permeate into the hydrophobic polymer. The enclosed drug particles absorb water, and at the end of the swelling process the matrices consist of an immense number of fluid pockets containing dissolved drugs. The effect of ultrasound on the release rates of these swollen matrices showed that low-frequency ultrasound $(20 \mathrm{kHz})$ reversibly increased the release rates from swollen matrices. The release rates of swollen matrices increased by a factor of 30-500, in contrast to the non-swollen matrices, where the release rates increased only by a factor of $2-3$. Non-swollen matrices contain drug particles which absorb ultrasound on the surface of the matrices, causing ultrasound attenuation. On the other hand, swollen matrices contain a compact arrangement of fluid pockets separated from each other by thin membranes containing a solution of the dissolved drug; therefore, ultrasound penetrates through the matrix volume. These findings are of clinical importance as they enable periodical treatments with controlled amounts of drug.

Magnetically-stimulated systems. Magnetically-stimulated systems involve drug molecules and magnetic beads which are uniformly distributed within a solid polymeric matrix. Although drug is released by diffusion when the device is exposed to fluids, a much higher release rate is obtained in the presence of an external oscillating magnetic field. Magnetically-responsive systems were characterized in vitro [38-41] and in vivo [42]. In vivo studies [42] showed that, when polymeric matrices made of ethylene-vinyl acetate copolymer containing insulin and magnetic beads were placed subcutaneously in diabetic rats for two months, glucose levels could be repeatedly and reproducibly decreased on demand by application of an oscillating magnetic field. The two primary parameters controlling the release rates in these systems are the magnetic field characteristics and the mechanical properties of the polymer matrix. It was found that when the frequency of the applied field was increased from 5 to $11 \mathrm{~Hz}$, the release rate of bovine serum albumin (BSA) from 
EVAc copolymer matrices rose in a linear fashion [40]. Saslavski et al. investigated the effect of magnetic field frequency and repeated field application on insulin release from alginate matrices and found that with repeated applications, inverse effects can occur: high frequencies gave a significant release enhancement for the second magnetic field application. Subsequent stimulation resulted in decreased enhancement due to the faster depletion at high frequencies. The mechanical properties of the polymeric matrix also affect the extent of magnetic enhancement [40]. For example, the modulus of elasticity of the EVAc copolymer can be easily altered by changing the vinyl acetate content of the copolymer. The release rate enhancement induced by the magnetic field increases as the modulus of elasticity of EVAc decreases.

Electrically-stimulated systems. The drug release in electrically controlled systems is triggered by the application of an electric field on a biodegradable polymer [43], rate-limiting membrane/hydrogel [44] and/or directly on the solute [45]. The electrophoretic migration of a charged macro solute within a hydrated membrane results from the combined response to the electrical forces on the solute and its associated counter-ions in the adjacent electrolyte solution [45]. Approaches involving micro electrical mechanical systems [46] or microchips [47-49] are being developed. Activation of each microchip reservoir can be controlled individually, creating a possibility for achieving many complex release patterns. Such a device has additional potential advantages including small size, quick response times and low power consumption. Carbon nanotubes [50] or polymeric biodegradable microchips [51] containing nano-size drug reservoirs might be able to deliver pharmaceuticals for long time periods in a controlled manner, by electrically opening the caps of these reservoirs. Electrically controlled membrane permeability has also been of interest in the field of electrically controlled or enhanced transdermal drug delivery [52].

Photo-stimulated systems. Photo-responsive polymers consist of a photoreceptor, usually a photochromic chromophore, and a functional part. The optical signal is captured by the photochromic molecules and then the isomerization of the chromophores in the photoreceptor converts it to a chemical signal. Photo-induced phase transition of gels $[49,53,54]$ and polymer degradation [55] have been also proposed for temporal drug delivery. Photo-responsive gels reversibly change their physical or chemical properties upon photo-radiation, thus affecting the release rate of drugs incorporated in the polymer. Lee et al. [56] demonstrated a new approach for an ophthalmic drug-delivery system. The principle of this approach is based on an azobenzene copolymer membrane (azo-polymer), which responds to various wavelengths depending on the substituents on the benzene ring. Azo-polymer film is coated on drug agents and deformed by polarized laser irradiation. Opened polymeric gate on azo-polymer film is closed by modulating the direction of polarization. Modifying the interference angle and polarization allows controlling the gating through the azo-polymer membrane and, therefore, controlling the drug release.

\section{Summary}

During past few years, polymer base targeting has become an important area of research and development and a considerable deal of work has been dedicated for developing stimuli sensitive molecules using smart polymers. Responsive polymeric drug delivery systems can target and adjusting drug release rates in response to internal or external stimulus. These smart polymers not only enhance the properties of drugs such as solubility, bioavailability, and prolonged circulation times, but also can be made to selectively release their cargo at the desired site of action. For the triggered release, these so-called smart drug delivery systems must be designed to react on application of a stimulus like $\mathrm{pH}$, temperature, redox potential, enzymes, light, ultrasound and magnetic field, as discussed in this review. These stimuli responsive polymers show a sharp change in properties upon a small or modest change in environmental condition, i.e., application of the stimuli. In recent years, many interesting biomedical uses have been proposed for stimuli-responsive polymers, such as application in diagnostics, drug delivery, tissue engineering, gene and protein 
delivery, etc. However, a few critical parameters are to be taken under consideration, such as biocompatibility and toxicology of these multi-component polymer-based systems, their ability to provide required levels of drug, and addressing necessary formulation issues in dosage design (e.g., shelf life, sterilization, reproducibility). While the successful development of polymeric targeting delivery systems is a significantly challenging job, these systems can provide considerable pharmacological benefits, which make this field an important and fruitful area for the future research [16].

\section{Acknowledgements}

The authors sincerely acknowledge the financial supports of the UGC, New Delhi under UPE-FARI Program [F.No. 14-3/2012 (NS/PE)] and the DST, New Delhi under DST-PURSE-Phase-II Program [F.No. SR/PURSE Phase 2/13(G)]. Furthermore, this research was supported by the European Regional Development Fund (FEDER), through COMPETE2020 under the PT2020 program (POCI-01-0145-FEDER-023423), and by the Portuguese Foundation for Science and Technology (UID/Multi/04044/2013) and PAMI (ROTEIRO/0328/2013; No 022158).

\section{References}

[1] N. V. Patil, Smart polymers are in the biotech future, BioProcess International, 4 (2006) 42-46.

[2] C. Alvarez-Lorenzo, A. Concheiro, Smart drug delivery systems: from fundamentals to the clinic, Chem. Commun., 50 (2014) 7743-7765.

[3] K. Modjarrad, S. Ebnesajjad, Handbook of Polymer Applications in Medicine and Medical Devices, first ed., William Andrew, Elsevier, 2013.

[4] H. R. James, R. John, A. Alex, K. R. Anoop, Smart polymers for the controlled delivery of drugs- a concise overview, Acta Pharmaceutica Sinica B, 4 (2014) 120-127.

[5] J. Chen, C. Chang, Fabrications and Applications of Stimulus-Responsive Polymer Films and Patterns on Surfaces: A Review, Materials, 7 (2014) 805-875.

[6] D. Schmaljohann, Thermo- and pH-responsive polymers in drug delivery, Adv. Drug Deliv. Rev., 58 (2006) 1655-1670.

[7] H. Almeida, M. H. Amaral, P. Lobão, Temperature and pH stimuli-responsive polymers and their applications in controlled and self-regulated drug delivery, J. Appl. Pharm. Sci., 02 (2012) 0110 .

[8] M. R. Aguilar, C. Elvira, A. Gallardo, B. Vázquez, J. S. Román, Smart Polymers and Their Applications as Biomaterials, in: N. Ashammakhi, R. Reis, E. Chiellini (Eds.), Topics in Tissue Engineering, Biomaterals, 2007, pp. 1-27.

[9] W. B. Liechty, D. R. Kryscio, B. V. Slaughter, N. A Peppas, Polymers for drug delivery systems, Ann. Rev. Chem. Biomol. Eng., 1 (2010) 149-173.

[10]M. A. Ward, T. K. Georgiou, Thermo responsive polymers for biomedical applications, Polymers, 3 (2011) 1215-1242.

[11]E. S. Gil, S. M. Hudson, Stimuli-reponsive polymers and their bioconjugates, Prog. Polym. Sci., 29 (2004) 1173-1222.

[12] S. Dai, P. Ravi, K. C. Tam, pH-responsive polymers: Synthesis, properties and applications, R. Soc. Chem. Soft Matter, 4 (2008) 435-449.

[13]J. Liu, Y. Huang, A. Kumar, A. Tan, S. Jin, A. Mozhi, X.J. Liang, pH-Sensitive nano-systems for drug delivery in cancer therapy, Biotechnol. Adv., 32 (2013) 693-710. 
[14] S. Talegaonkar, L. M. Negi, H. Sharma, Sobiya Zafar, Smart polymers in targeted drug delivery, in: V. K. Thakur, M. K. Thakur (Eds.), Handbook of Sustainable Polymers structure and Chemistry, CRC Press, Taylor and Francis Group, Pan Stanford, 2016, pp. 737-816.

[15]H. Wei, R.X. Zhuo, X.-Z. Zhang, Design and development of polymeric micelles with cleavable links for intracellular drug delivery, Prog. Polym. Sci., 38 (2013) 503-535.

[16]R. V. Ulijin, Enzyme-responsive materials: A new class of smart biomaterials, J. Mater. Chem., 16 (2006) 2217-2225.

[17] J. Hu, G. Zhang, S Liu, Enzyme-responsive polymeric assemblies, nanoparticles and hydrogels, Chem. Soc. Rev., 41 (2012) 5933-5949.

[18] N. Song, W. Liu, Q. Tu, R. Liu, Y. Zhang, J. Wang, Preparation and in vitro properties of redox-responsive polymeric nanoparticles for paclitaxel delivery, Colloids Surf. B, 87 (2011) 454 463.

[19]M. Li, Stimuli-responsive polymer-protein conjugates prepared by reversible additionfragmentation chain transfer polymerization, Dissertation, Southern Methodist University, 3418903 (2010) 185.

[20] M. Irie, Photoresponsive polymers, Adv. Polym. Sci., 94 (1990) 27- 67.

[21] O. Pieroni, A. Fissi, N. Angelini, F. Lenci, Photoresponsive polypeptides, Acc. Chem. Res., 34 (2001) 9-17.

[22]T. Shimoboji, E. Larenas, T. Fowler, S. Kulkarni, A. S. Hoffman, P. S. Stayton, Photoresponsive polymer-enzyme switches, PNAS, 99 (2002) 16592-16596.

[23] J. Thévenot, H. Oliveira, O. Sandre, S. Lecommandoux, Magnetic responsive polymer composite materials, Chem. Soc. Rev., 42 (2013) 7099-7116.

[24]B. Luo, X. J. Song, F. Zhang, A. Xia, W. L. Yang, J. H. Hu, C. C. Wang, Multi-functional thermosensitive composite microspheres with high magnetic susceptibility based on magnetite colloidal nanoparticle clusters, Langmuir, 26 (2010) 1674-1679.

[25]A. K. Gupta, R. R. Naregalkar, V. D. Vaidya, M. Gupta, Recent advances on surface engineering of magnetic iron oxide nanoparticles and their biomedical applications, Nanomedicine, 2 (2007) 23-39.

[26] E. Fleige, M. A. Quadir, R. Haag, Stimuli-responsive polymeric nanocarriers for the controlled transport of active compounds: Concepts and applications, Adv. Drug Deliv. Rev., 64 (2012) 866884.

[27] Information on http://depts.washington.edu/chem/people/faculty/boydston.html

[28]D. Bennet, S. Kim, Application of Nanotechnology in Drug Delivery, in: A. Demir Sezer (Eds.), Polymer Nanoparticles for Smart Drug Delivery, InTech, 2014, pp.257-310.

[29]J. Kost and R. Langer, Responsive polymeric delivery systems, Adv. Drug Deliv. Rev., 46 (2001) 125-148.

[30] J. Kopecek, Smart and genetically engineered biomaterials and drug delivery systems, Eur. J. Pharm. Sci., 20(2003) 1-16.

[31]D. Schmaljohann, Thermo- and pH-responsive polymers in drug delivery, Adv. Drug Deliv. Rev., 58 (2006) 1655-1670.

[32] C. Alexander, Temperature- and pH-responsive smart polymers for gene delivery, Expert. Opin. Drug Deliv., 3 (2006)573-581. 
[33] C. Wang, R. J. Stewart, J. Kopecek, Hybrid hydrogels assembled from synthetic polymers and coiled-coil protein domains, Nature, 397 (1999) 417-420.

[34]D. L. H. Alarcon C, S. Pennadam, C. Alexander, Stimuli responsive polymers for biomedical applications, Chem. Soc. Rev., 34 (2005) 276-285.

[35] R. M. K. Ramanan, P. Chellamuthu, L. Tang, K. T. Nguyen, Development of temperature sensitive composite hydrogel for drug delivery applications, Biotechnol. Prog., 22 (2006) 118-125.

[36]A. M. Lowman, N. A. Peppas, Pulsatile Drug Delivery Based on a Complexation/Decomplexation Mechanism, ACS Symposium Series, 728 (1999) 30-42.

[37] T. Traitel, R. Goldbart, J. Kost, Smart polymers for responsive drug-delivery systems, J. Biomater. Sci. Polymer Edn., 19 (2008) 755- 767.

[38] J. Kost, K. Leong, R. Langer, Ultrasound-enhanced polymer degradation and release of incorporated substances, Proc. Natl. Acad. Sci. USA, 86 (1989) 7663-7666.

[39] C. Aschkenasy, J. Kost, On-demand release by ultrasound from swollen hydrophobic matrices, J. Control. Rel., 110 (2005) 58-66.

[40]E. Edelman, J. Kost, H. Bobeck, R. Langer, Regulation of drug release from polymer matrices by oscillating magnetic fields, J. Biomed. Mater. Res., 19 (1985) 67-83.

[41]D. S. T. Hsieh, R. Langer, J. Folkman, Magnetic modulation of release of macromolecules from polymers, Proc. Natl. Acad. Sci. USA, 78 (1981) 1863-1867.

[42] J. Kost, R. Noecker, E. Kunica, R. Langer, Magnetically controlled release systems: effect of polymer composition, J. Biomed. Mater. Res., 19 (1985) 935-40.

[43]E. R. Edelman, L. Brown, J. Taylor, R. Langer, In vitro and in vivo kinetics of regulated drug release from polymer matrices by oscillating magnetic fields, J. Biomed. Mater. Res., 21(1987) 339353.

[44]J. Kost, J. Wolfrum, R. Langer, Magnetically enhanced insulin release in diabetic rats, J. Biomed. Mater. Res., 21(1987) 1367-1373.

[45] O. Saslavski, P. Couvrer, N. A. Peppas, Controlled Release of Bioactive Materials, in: J. Heller, F. Harris, H. Lohmann, H. Merkle, J. Robinson (Eds), Controlled Release Society, Basel, (1988), pp. 26.

[46] I. Kwon, Y. Bae, S. Kim, Electrically credible polymer gel for controlled release of drugs, Nature, 354 (1991) 291-293.

[47]H. Xu, C. Wang, C. Wang, J. Zoval, M. Madou, Polymer actuator valves toward controlled drug delivery application, Biosens. Bioelectron. 21 (2006) 2094-9.

[48]A. J. Grodzinsky, P. E. Grimshaw, Pulsed and Self-Regulated Drug Delivery, in: J. Kost (Ed.), CRC Press, Boca Raton, FL, 1990, pp. 47.

[49] I. Galaev, B. Mattiasson, Smart polymers and what they could do in biotechnology and medicine, Trends Biotechnol., 17 (1999) 335-340.

[50]D. LaVan, T. McGuire, R. Langer, Small-scale systems for in vivo drug delivery, Nature Biotechnol., 21 (2003) 1184-1191.

[51] J. T. Santini, M. J. Cima, R. Langer, A controlled-release microchip, Nature, 397(1999) 335338.

[52]J. West, Drug delivery: Pulsed polymers, Nature Mater., 2 (2003) 709-710. 
[53]A. Bianco, K. Kostarelos, M. Prato, Applications of carbon nanotubes in drug delivery, Curr. Opin. Chem. Biol., 9 (2005) 674-679.

[54]A. Richards Grayson, I. Choi, B. Tyler, P. Wang, H. Brem, M. Cima, R. Langer, Multi-pulse drug delivery from a resorbable polymeric microchip device, Nature Mater., 2 (2003)767-772.

[55] M. Prausnitz, S. Mitragotri, R. Langer, Current status and future potential of transdermal drug delivery, Nature Rev. Drug Discov., 3 (2004) 115-124.

[56] A. Mamada, T. Tanaka, D. Kungwatchakun, M. Irie, Photoinduced phase transition of gels, Macromolecules, 23 (1990) 1517-1519.

[57]A. Suzuki, T. Tanaka, Phase transition in polymer gels induced by visible light, Nature, 346 (1990) 345-347.

[58]N. Yui, T. Okano, Y. Sakurai, Photo-responsive degradation of heterogeneous hydrogels comprising crosslinked hyaluronic acid and lipid microspheres for temporal drug delivery, J. Control. Rel., 26 (1993) 141-145.

[59] J. K. Lee, H. Lee, E. Jang, S.-D. Lee, S. J. Kim, Photo triggering of the membranes gate in photo-responsive polymer for drug release, Engineering in Medicine and Biology Society, 27th Annual International Conference of the IEEE-EMBS, (2005), pp. 5069-5072. 